\title{
REVIEW
}

\section{Lactic acidosis in HIV infected patients: a systematic review of published cases}

\author{
A Arenas-Pinto, A D Grant, S Edwards, I V D Weller
}

Sex Transm Infect 2003;79:340-344

See end of article for authors' affiliations

.....................

Correspondence to: Dr Alejandro Arenas-Pinto, Department of Sexually Transmitted Diseases, Royal Free and University College Medical School, University College London, The Mortimer Market Centre, Mortimer Market, off Capper Street, London WCIE 6AU, UK:

AArenas-Pinto@ gum.ucl.ac.uk

Accepted for publication 6 February 2003

\begin{abstract}
Objective: To describe the clinical, epidemiological, and biochemical characteristics of published cases of lactic acidosis (LA) and to generate hypotheses concerning risk factors associated with this complication.

Methods: Systematic review of cases reported in the medical literature.

Results: 217 published cases were identified, 90 of which fulfilled the study definition and had sufficient individual data on potential risk factors to be included. The 90 patients had a mean age of 40.1 years (range 16-69) and 53\% were female. All 90 patients were taking nucleoside reverse transcriptase inhibitors (NRTI) at the time of the episode. Among the 83 patients with details of their antiretroviral therapy (ART) regimen 51 patients were taking stavudine, 29 zidovudine, 27 didanosine, and 25 lamivudine. Around $50 \%$ of the patients had abdominal pain, nausea, or vomiting. Hepatic steatosis was consistently reported (53/90) and in $36(68 \%)$ there was histological evidence. The case fatality rate was $48 \%$. Six cases were rechallenged with NRTI and three developed a further LA episode. Using data on the numbers of HIV infected individuals receiving care in the United States, we estimate that the risk of LA could be 2.5 times higher for women than men.

Conclusions: NRTI use and female sex appear to be risk factors for the development of LA. What other factors are involved is still not clear but might include duration of NRTI therapy, specific drug use, and genetic predisposition. A case-control study is needed to better define risk factors for severe LA.
\end{abstract}

tion actic acidosis (LA) is an uncommon but serious complication of antiretroviral therapy (ART). The reported inciLdence rates vary from 1.3 to 10 per 1000 person years on $\mathrm{ART}^{1-4}$; one explanation for this may be differences in case definition. Asymptomatic hyperlactataemia is a common finding. The prevalence of hyperlactataemia in outpatients on ART is around $9-16 \% .{ }^{5}$ Among untreated patients it is about $2 \%{ }^{7}$ However, only $1 \%$ of those with raised lactate levels have severe hyperlactataemia (lactate $>5 \mathrm{mmol} / \mathrm{l}$ ). ${ }^{57}$ Between asymptomatic hyperlactataemia and severe lactic acidosis there is a group of patients with moderately raised lactate (2.5-5 $\mathrm{mmol} / \mathrm{l})$ and non-specific symptoms referred to as symptomatic hyperlactataemia with an incidence rate estimated to be between 8 to 20.9 cases per 1000 person years at risk. ${ }^{3} 8$

Studies to date suggest that LA is caused by mitochondrial toxicity through the inhibition of mitochondrial DNA polymerase $\gamma$ (mtDNA polymerase $\gamma$ ) by nucleotide reverse transcriptase inhibitors (NRTI). In vitro studies have shown that NRTI vary in their ability to inhibit mtDNA polymerase $\gamma$ with a hierarchy of effect. Drugs such as stavudine (d4T), didanosine (ddI), and zalcitabine ( $\mathrm{ddC}$ ) induce more mtDNA inhibition than others. ${ }^{10-12}$ There is evidence of variable tissue specificity, determined by differences in cellular penetration and phosphorylation, different mitochondrial uptake of NRTI and their metabolites, and the tissue dependence on mitochondrial metabolism. ${ }^{10}$

\section{METHODS}

Published cases of severe LA in HIV infected adults were reviewed: Medline (1982-2000), Aidsline (1982-2001), Web of Science, the Cochrane Database of Systematic Reviews (Issue 2, 2001), and the CAB Abstract Database. The key terms used for the search were lactic acid*, metabolic acid*, and HIV infection for Medline and lactic acidosis, lactic acidaemia, metabolic acidosis, metabolic acidaemia, and hepatic steatosis for Aidsline. Abstracts of relevant conferences were reviewed as indexed references on Aidsline and in available abstract books of relevant scientific events such as international AIDS conferences and workshops. All references cited in included papers were checked and included if pertinent. Reports from clinical trials, which recorded drug adverse events, were not searched.

Cases were included if the patient was over 16 and was hospitalised with biochemical evidence of LA (one or more of the following: $\mathrm{pH}<7.34$, serum bicarbonate $<20 \mathrm{mmol} / \mathrm{l}$, anion gap $>12 \mathrm{mEq} / \mathrm{l}$ ). If biochemical data were not available, the patient was included when the author(s) stated the case had lactic acidosis. Patients who had other underlying potential causes for LA were excluded.

Date, author, city, and hospital of the report were checked for duplication of reporting. History of HIV related complications, previous medical history, clinical symptoms and signs, laboratory results, and evidence for mitochondrial toxicity were also collected onto a standardised proforma.

Continuous variables were summarised using the mean and standard deviation if normally distributed or median and range if not. For categorical data, variables were presented as proportions.

\section{RESULTS}

The review identified 217 cases of LA; 87 were presented as part of case series with aggregated data without sufficient individual data on potential risk factors to be included; 40 were reported as part of cohort studies examining outcomes other than LA without individual descriptions. The remaining 90 fulfilled our definition and were included in this study.

\section{Demographic data}

The mean age of the 90 patients was 40 years (range 16-69 years), 47 (53\%) were female. Three of the female patients were pregnant, all at 32 weeks or more of gestation at presentation. Reports from three countries account for the $83 \%$ of the 


\begin{tabular}{|c|c|c|}
\hline ART & Cases & $\%$ \\
\hline Monotherapy & 30 & \\
\hline Zidovudine & 26 & 87 \\
\hline Didanosine & 2 & 7 \\
\hline Stavudine & 2 & 7 \\
\hline 2 drugs & 16 & \\
\hline Zidovudine + didanosine & 2 & 13 \\
\hline Ziduvudine + stavudine & 1 & 6 \\
\hline Stavudine + didanosine & 6 & 38 \\
\hline Stavudine + lamivudine & 7 & 44 \\
\hline 3 or more drugs & 37 & \\
\hline Stavudine + didanosine + lamivudine & 1 & 3 \\
\hline Stavudine + didanosine + PI & 12 & 32 \\
\hline Stavudine + lamivudine + PI & 12 & 32 \\
\hline Stavudine + didanosine + nevirapine & 4 & 11 \\
\hline Stavudine + lamivudine + nevirapine & 3 & 8 \\
\hline Stavudine + lamivudine + delavirdine & 1 & 3 \\
\hline Stavudine + didanosine + efavirenz & 1 & 3 \\
\hline Stavudine + didanosine + delavirdine $+\mathrm{Pl}$ & 1 & 3 \\
\hline Stavudine + lamivudine + nevirapine + Pl & 1 & 3 \\
\hline Stavudine + 2 Pl & 1 & 3 \\
\hline
\end{tabular}

included cases; 56 (62\%) from the United States, 14 (16\%) from France, and five (6\%) from the Netherlands.

\section{ART at the time of the LA event}

ART at the time of the event was recorded in 83 of the 90 patients (table 1). Information about past ART history before the LA episode was sparse. All cases were taking at least one NRTI at the time of the LA episode. Thirty cases (36\%) were on monotherapy regimens when LA was diagnosed, 26 were on zidovudine (ZDV), 16 (19\%) were taking two ART drugs (a combination of NRTI), and 37 (43\%) were on combinations of three or more drugs, including either a protease inhibitor (PI) or NNRTI, at the time of the LA event. One additional patient was taking three NRTI.

Fifty one of $83(61 \%)$ patients were taking d4T, 49 of them with at least one other antiretroviral drug. The median duration of d4T treatment was 8 (range 1-36) months (data available for 45/51 patients); 29/83 (35\%) cases were on ZDV for a median of 8 (range 1-24) months (data from 25/29 cases).

Twenty seven of 83 cases (33\%) were on ddI for a median of 8.5 (range 3-48) months (data from 23/27 patients); 25/83 patients $(30 \%)$ were on lamivudine (3TC) for a median of 8 (range $1-18)$ months (23/25 cases). No patient was on abacavir $(\mathrm{ABC})$ or zalcitabine (ddC) at the time LA was diagnosed. In 17 patients with previous ART regimens reported, seven had received ddC; 13 of these 17 cases were on d4T at the time of the LA event, but all of them had received ZDV therapy in the past.

Patients on ZDV at the LA event had more advanced disease than those exposed to other NRTI. The median CD4 cell count among patients on ZDV was $79 \times 10^{6} / 1$ (range 18-406) (data from 10/29 cases) compared to $282.5 \times 10^{6} / 1$ (range 35-1321) (data from 36/61 cases) in those not on ZDV.

Previous adverse effects related to ART were described in 16 patients, and the majority could have been attributed to NRTI toxicity (six cases of myelosuppression, four cases of myopathy, four cases of peripheral neuropathy, and one case of pancreatitis). One patient had lipoatrophy.

\section{Past medical history and previous ART related adverse effects}

In 17 cases previous hepatic diseases were mentioned and 11 of these were viral hepatitis; two had chronic hepatitis B
Table 2 Symptoms reported at the time of the lactic acidosis event

\begin{tabular}{lll}
\hline Symptom & $\begin{array}{l}\text { Number of cases } \\
(\mathbf{n}=85)\end{array}$ & $\begin{array}{l}\text { Proportion } \\
(\%)\end{array}$ \\
\hline Nausea & 45 & 53 \\
Vomiting & 44 & 52 \\
Abdominal pain & 38 & 45 \\
Weight loss & 19 & 22 \\
Weakness & 19 & 22 \\
Dyspnoea & 19 & 22 \\
Anorexia & 16 & 19 \\
Tachypnoea & 13 & 15 \\
Diarrhoea & 8 & 9 \\
Fatigue & 7 & 8 \\
Abdominal distension & 5 & 6 \\
Impairment of consciousness & 2 & 2 \\
Other & 8 & 9 \\
\hline
\end{tabular}

(HBsAg positive), two hepatitis C (HCV RNA positive), four previous acute hepatitis $\mathrm{B}$, one past hepatitis $\mathrm{A}$. There was no specific information on the two other patients with a history of hepatitis.

Among only 24 patients with information about concurrent drug therapy other than ART, nine were on concurrent treatment with potentially hepatotoxic drugs such as fluconazole, ketaconazole, or isoniazid.

\section{Symptoms and laboratory findings at the time of LA event}

Non-specific gastrointestinal symptoms; nausea, vomiting, and abdominal pain were the most frequently reported, in over $50 \%$ of the 85 patients with available information (table 2 ). Dyspnoea and tachypnoea classically associated with metabolic acidosis were reported in 35 (41\%).

Among 79 patients in whom blood lactate levels were reported, the median was 10.5 (range $2.4-168.5$ ) $\mathrm{mmol} / \mathrm{l}$. The anion gap was described in 32 patients, median 25.5 (range 10-42) $\mathrm{mEq} / \mathrm{l}$ (table 3 ). The values on the day of admission were used even if subsequent values were more abnormal. For this reason some of the ranges include normal values. There was laboratory evidence of mild to moderate hepatic dysfunction in 41 of the 63 cases $(65 \%)$ in whom information was given, with median transaminase values between 1.5 and 2.5 times over the upper limit of normal (range 1.4-10.7 times over the upper limit of normal).

In 39 premortem or necropsy liver biopsies 36 (92\%) had hepatic steatosis; $12 / 39$ (31\%) macrovesicular steatosis, 8/39 (21\%) microvesicular steatosis, and 16/39 (41\%) a mixed pattern. The other three biopsies showed hepatic fibrosis and inflammation.

In 11 patients mitochondrial morphology or function were examined. In nine patients electron microscopy was performed on liver or muscle biopsies or both. In four who had only liver biopsies, enlargement, alteration of shape, or increased mitochondrial density were reported. ${ }^{8}{ }^{13-15}$ In three patients who had muscle biopsies two showed similar mitochondrial ultrastructural changes ${ }^{16}$ and one was reported as normal. ${ }^{18}$ In one patient who had both a liver biopsy and muscle biopsy, the liver biopsy was abnormal but the muscle biopsy was normal. The remaining patient had a normal muscle biopsy. ${ }^{19}$

Respiratory chain dysfunction was examined in only three cases using spectrophotometric determination of enzymatic complex activity. MtDNA depletion and particularly low levels of activity of complexes 2, 3, and 4 was reported in one case using muscle mitochondria. ${ }^{20}$ Two other patients with biochemical evidence of mitochondrial dysfunction were tested using hepatic mitochondria and had reduction of complexes 1 and $1,3,4$, and 5 respectively. ${ }^{13}{ }^{21}$ Finally, mtDNA 
Table 3 Acid base status of the patients at the time of the lactic acidosis event

\begin{tabular}{lllll}
\hline Test & Normal range & No & Median & Range \\
\hline Lactate $(\mathrm{mmol} / \mathrm{l})$ & $0.7-1.2$ & 79 & 10.5 & $2.4-168.5$ \\
$\mathrm{pH}$ & $7.35-7.45$ & 52 & 7.2 & $6.67-7.42$ \\
Bicarbonate $(\mathrm{mmol} / \mathrm{l})$ & $24-29$ & 51 & 8 & $1.2-26$ \\
Anion gap $(\mathrm{mEq} / \mathrm{l})$ & $8.0-12.0$ & 32 & 25.5 & $10.0-42.0$ \\
\hline
\end{tabular}

Table 4 Estimated relative risk of developing lactic acidosis by sex

\begin{tabular}{|c|c|c|c|c|c|}
\hline & $\begin{array}{l}\text { Total HIV infected } \\
\text { patients* }\end{array}$ & $\begin{array}{l}\text { Patients with CD4 } \\
<500 \times 10^{6} / /\end{array}$ & $\begin{array}{l}\text { Lactic acidosis cases } \\
\text { observed }\end{array}$ & $\begin{array}{l}\text { Lactic acidosis cases } \\
\text { expected } \dagger\end{array}$ & $R R$ \\
\hline Female & 52200 & 44892 & 30 & 12 & 2.5 \\
\hline Male & 179200 & 164864 & 26 & 44 & \\
\hline Total & 231400 & 209756 & 56 & & \\
\hline
\end{tabular}

*From Bozzette, et al. ${ }^{30}$

tIf risk of LA were the same for men and women.

depletion was reported in one other patient on muscle and liver biopsy but enzymatic complexes were not examined. ${ }^{22}$

\section{Outcome}

Forty three of $90(48 \%)$ patients died in a median period of 7 days (range 2 days to 30 weeks) after diagnosis. Among the 46 survivors, the median time of follow up was 3 months (range $0.25-30$ months). In $13 / 46(28.3 \%)$ cases it was stated that ART was restarted, using NRTI in six of these patients, but the exact duration of follow up was reported only in three of these six. Three of six patients rechallenged with NRTI had further episodes of LA. Two were females. One male and one female were challenged with ZDV as monotherapy; both developed a further episode of LA but no other adverse effect attributable to NRTI. ${ }^{23}{ }^{24}$ The other female's re-challenge regimen was not specified. An additional female patient was re-treated with a combination of 3TC and nelfinavir and followed for 26 months, with no further LA or other adverse event. ${ }^{18}$ One male patient who developed LA on a combination of d4T and ddI did not resume ART. However, 22 months after discontinuation he developed fatal portal hypertension, liver failure, and hepatic mitochondrial dysfunction with normal serum lactate. ${ }^{13}$

\section{DISCUSSION}

This is the largest review of published cases of LA to date. Since only 90 cases had sufficient details to be included, robust conclusions about the possible risk factors associated with LA are not possible. However, the results do add some weight to proposed risk factors from the individual case reports, case series, and other smaller reviews. Mégarbane et al described 40 cases published during the 1990s accompanied by a literature review of possible causes. ${ }^{25}$ Falcó et al reviewed 60 cases, and performed a multivariate analysis of possible prognostic factors for mortality. ${ }^{26}$ Neither of these reviews aimed to identify risk factors for LA and their literature review strategies were not described.

Females appear to be at greater risk of developing LA. ${ }^{27} 28$ Our case reports were predominantly from the United States, Western Europe, and Australia, and more cases were female. In all these areas the majority of HIV infected patients (75-90\%) are male, ${ }^{29}$ so this could represent a relatively higher risk of LA among women.

Using data on the numbers of HIV infected individuals receiving care in the United States, ${ }^{30}$ we made a very approximate estimate of the denominator of patients receiving ART, from the estimated number of patients with less than 500 CD4 cells $\times 10^{6} / 1$. Of the total of 209756 patients likely to be exposed to ART, 44892 (21\%) were female. Assuming no sex bias in publication of cases, if there were no association between sex and LA, among the 56 US cases, the expected number of females would be 11 , but the observed number was 30. These data suggest that the risk of developing LA could be 2.5 times higher in women than in men (table 4).

Combination therapy is not randomly allocated, patients switch treatments and have long ART histories which are poorly recorded. It is therefore difficult to estimate the contribution of individual drugs to a toxicity, particularly if the event is the result of cumulative exposure to a drug class rather than an individual drug exposure. A background of HIV infection is not required for NRTI to produce LA, as there are reports of LA in HIV negative neonates exposed to prophylactic ZDV. ${ }^{31}{ }^{32}$

ZDV associated LA events were reported mainly during the monotherapy era. ${ }^{25}$ In our series, $90 \%$ of those cases on ZDV at the time of the LA episode were taking it as monotherapy. The cases of LA associated with ZDV therapy had more advanced disease compared with patients exposed to NRTI other than ZDV. Additionally, the case fatality rate (CFR) among ZDV treated patients was $68 \%$, compared with $37 \%$ for patients not exposed to ZDV. These differences probably reflect the use of ZDV monotherapy in patients with more advanced HIV disease in the late 1980s and early 1990s.

The association between d4T use and hyperlactataemia or LA has been highlighted in previous studies. ${ }^{21}{ }^{33}$ However, 51 of the 53 patients on $\mathrm{d} 4 \mathrm{~T}$ in our review were taking it as part of combination therapy, which included at least one additional NRTI. Cumulative toxicity has been proposed as a risk factor for mitochondrial toxicity and the total duration of exposure to NRTI could be important. ${ }^{34}$ The median NRTI exposure of patients included in our review was 8 months, but in most cases this period refers to the regimen on which LA occurred and not the total time exposed to NRTI. If cumulative toxicity is important ${ }^{172435}$ some of the association of LA with d4T might be confounded by a long duration of NRTI exposure. Previous ART regimens were reported in only 13 of the 52 patients on $\mathrm{d} 4 \mathrm{~T}$ and all of them had a previous history of ZDV use. Carr et al found that an important proportion of patients on $\mathrm{d} 4 \mathrm{~T}$ had received previous treatment with $\mathrm{ZDV}^{36}$ By contrast, John et al reported that the association between $\mathrm{d} 4 \mathrm{~T}$ and hyperlactataemia was not confounded by previous treatment with ZDV. ${ }^{3}$ The contribution of other NRTI to the development of LA events is difficult to evaluate because the majority of patients taking ddI or 3TC $(92 \%)$ were also taking d4T. Lonergan et al recently reported improvements in both laboratory abnormalities and clinical symptoms when d4T was replaced by either ABC or ZDV in symptomatic patients with hyperlactataemia. ${ }^{37}$ 


\section{Key messages}

- Female patients might be at a greater risk of developing lactic acidosis than males, once they are exposed to ART

- The total exposure to NRTI might be an important variable, but this is poorly reported in case reports and case series

- Hepatic steatosis and mild to moderate elevation in liver function test are frequently reported in patients with lactic acidosis. The implications of these findings are not well understood to date

- Mitochondrial toxicity is believed to be the cause of lactic acidosis in HIV infected patients exposed to NRTI; however the number of cases that have had studies of mitochondrial morphology or function are small.

This review also highlights that a common presentation of LA is with non-specific gastrointestinal symptoms. A high level of suspicion is required if an early diagnosis is to be made.

Previous reviewers have reported high case-fatality ratio (CFR) associated with LA, of around $60 \%,{ }^{25}{ }^{35}$ but in a more recent study it was $33 \%{ }^{26}$ In our review CFR was $48 \%$ overall. In the first 45 cases, published between 1991 and 1999, the CFR was $64 \%$. In contrast, for the second 45 cases, published between 2000 and June 2001, it was 33\%. The lower CFR among more recently published cases might be due to a higher level of awareness among clinicians about LA and earlier identification of cases.

In our review, only a small number of cases had investigation of mitochondrial morphology or function. At least 43 studies have shown evidence of NRTI related mitochondrial toxicity in vitro, and in animal models. ${ }^{10}$ Most of the evidence for mtDNA depletion in clinical studies has been in cases of ZDV related myopathy. ${ }^{38}$

The proposed mechanism for LA is inhibition of mtDNA polymerase $\gamma$ leading to mtDNA depletion. Walker et al showed that ZDV and the combination ZDV/3TC can produce raised lactate levels and cell death without a reduction in or deletions of mtDNA in an in vitro study. ${ }^{39}$ Bartley et al have reported multiple hepatic mtDNA deletions in a patient with LA. ${ }^{40}$ Recently we have described a case of LA with marked reduction in respiratory chain enzyme activity but not mtDNA depletion and direct NRTI induced toxicity on the respiratory chain has also been proposed. ${ }^{41}{ }^{42}$

Mitochondrial dysfunction would be expected to lead to an increase in endogenous lactate production. Leclerq et al, using an exogenous lactate challenge test in 11 symptomatic and three asymptomatic patients with hyperlactataemia, showed a marked increase in endogenous lactate production even in patients with a moderate increase in serum lactate. ${ }^{43}$ In addition, in the face of this overproduction of lactate, factors which affect lactate clearance such as hepatic dysfunction, might also be expected to contribute to hyperlactataemia and lactic acidosis. ${ }^{44}$ Concomitant use of drugs such as ribavirin with other NRTI-that is, ddI, has also proposed as a risk factor to develop mitochondrial dysfunction and LA but in patients with hepatic dysfunction caused by hepatitis C. ${ }^{45}$

In summary, LA is likely to be an NRTI related adverse effect, which affects female patients more frequently. It is difficult to evaluate the precise role of individual NRTI drugs from case reports. It is possible that $\mathrm{d} 4 \mathrm{~T}$, and probably also ZDV, may be more likely to induce LA than other NRTI. Since the clinical presentation of LA is frequently non-specific and because raised levels of lactate occur in asymptomatic patients, a standard case definition is required to assess risk factors and prognostic factors accurately. A large case-control study is needed to better define risk factors for severe LA.

\section{ACKNOWLEDGEMENTS}

AAP is funded by the Consejo de Desarrollo Científico y Humanístico de la Universidad Central de Venezuela. We thank Professor Stephen
Evans (London School of Hygiene and Tropical Medicine) for helpful comments on this manuscript.

\section{CONTRIBUTORS}

AAP performed the systematic review and wrote the first and final drafts of the manuscript; ADG reviewed the searching strategy, critically reviewed the data analysis and the manuscript; SE critically reviewed the proforma used to collect cases information and the manuscript drafts; IVDW proposed the project, reviewed the analysis strategy, and assisted in the writing of the manuscript.

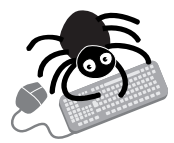

Further references can be found on the STI website (www.stijournal.com/supplemental)

\section{Authors' affiliations}

A Arenas-Pinto, I V D Weller, Department of Sexually Transmitted Diseases, Royal Free and University College Medical School. University College London, UK

A D Grant, Clinical Research Unit, London School of Hygiene and Tropical Medicine

A Arenas-Pinto, S Edwards, I V D Weller, Camden Primary Care Trust, The Mortimer Market Centre

\section{REFERENCES:}

1 Fortgang IS, Belitsos PC, Chaisson RE, et al. Hepatomegaly and steatosis in HIV-infected patients receiving nucleoside analog antiretroviral therapy. Am J Gastroent 1995;90:1433-6

2 Brinkman K, Troost N, Schrijnders L, et al. Usefulness of routine lactate measurement to prevent lactic acidosis: evaluation of a protocol. 9th Conference on Retroviruses and Opportunistic Infections. Seattle 2002 [abstract?.

3 John M, Moore CB, James IR, et al. Chronic hyperlactatemia in HIV-infected patients taking antiretroviral therapy. AIDS 2001;15:717-23

4 Gérard Y, Ajana F, Yazdanpanah Y, et al. Hyperlactatemia in 59 patients during a 4-year followed-up cohort study: implications for routine lactate monitoring. 3rd International Workshop on Adverse Drug Reactions and Lipodystrophy in HIV. Athens 2001 [abstract 65].

5 Vrovenraets S, Treskes $M$, Regez RM, et al. The occurrence of hyperlactatemia in HIV-infected patients on NRTI treatment. XIII International AIDS Conference. Durban 2000 [abstract TuPpB 1234]

6 Datta D, Mandalia S, Morlese J, et al. Biochemical abnormalities associated with hyperlactataemia in HIV-1 positive patients. Ist IAS Conference on HIV Pathogenesis and Treatment. Buenos Aires, July 2001. [abstract 518].

7 Moyle GJ, Datta D, Mandalia S, et al. Hyperlactataemia and lactic acidosis during antiretroviral therapy:relevance, reproducibility and possible risk factors. 3rd International Workshop on Adverse Drug Reactions and Lipodystrophy in HIV. Athens 2001 [abstract 98]

8 Lonergan JT, Behling C, Pfander $\mathrm{H}$, et al. Hyperlactatemia and hepatic abnormalities in 10 human immunodeficiency virus-infected patients receiving nucleoside analogue combination regimens. Clin Infect Dis 2000;31:162-6.

9 Gérard Y, Maulin L, Yazdanpanah Y, et al. Symptomatic hyperlactataemia: an emerging complication of antiretroviral therapy. AIDS 2000;14:2723-30.

10 Kakuda, TN. Pharmacology on nucleoside and nucleotide reverse transcriptase inhibitor-induced mitochondrial toxicity. Clin Ther 2000;22:685-708

11 Moyle G. Clinical manifestations and management of antiretroviral nucleoside analog-related mitochondrial toxicity. Clin Ther 2000;22:911-36

12 Brinkman K, Kakuda T. Mitochondrial toxicity of nucleoside analogue reverse transcriptase inhibitors:a looming obstacle for long-term antiretroviral therapy? Curr Opin Infect Dis 2000;13:5-11

13 Carr A, Morey A, Mallon P, et al. Fatal portal hypertension, liver failure and mitochondrial dysfunction after HIV-1 nucleoside analogue-induced hepatitis and lactic acidaemia. Lancet 2001 May 5;357:1412-4

14 Charton-Bain MC, Flamant M, Aubertin JM, et al. Acidose lactique, et altérations mitochondriales hépatiques au cours d'un traitement par zidovudine. Gastroenterol Clin Biol 1997;21:979-81

15 Khouri S, Cushing $\mathrm{H}$. Lactic acidosis secondary to nucleoside analog antiretroviral therapy. Infect Med 2000;17:547-54

16 Gopinath R, Hutcheon M, Cheema-Dhadli S, et al. Chronic lactic acidosis in a patient with acquired immunodeficiency syndrome and mitochondrial myophathy:biochemical studies. J Am Soc Nephrol 1992;3:1212-9.

17 Sundar K, Suarez M, Banogon P, et al. Zidovudine-induced fatal lactic acidosis and hepatic failure in patients with acquired immunodeficiency syndrome: report of two patients and review of the literature. Crit Care Med 1997;25: 1425-30. 
18 Shaer AJ, Rastegar A. Lactic acidosis in the setting of antiretroviral therapy for the acquired immunodeficiency syndrome. Am J Nephrol 2000;20:332-8

19 Olano JP, Borucki M, Wen JW, et al. Massive hepatic steatosis and lactic acidosis in a patient with AIDS who was receiving zidovudine. Clin Infect Dis 1995:21:973-6.

20 Roy PM, Gouello JP, Pennison-Besnier I, et al. Severe lactic acidosis induced by nucleoside analogues in an HIV-infected man. Ann Emerg Med 1999;34:282-4

21 Brivet FG, Nion I, Mégarbane B, et al. Fatal lactic acidosis and liver steatosis associated with didanosine and stavudine treatment:a respiratory chain dysfunction? J Hepatol 2000;32:364-5.

22 Chairot P, Drogou I, Lacroix-Szmania I, et al. Zidovudine-induced mitochondrial disorder with massive liver steatosis, myopathy, lactic acidosis and mitochondrial DNA depletion. J Hepatol 1999:30:156-60

23 Coyle T, Abel E. Lactic acidosis and AIDS (letter). Ann Intern Med 1993; 19:344

24 Freiman JP, Helfert KE, Hamrell MR, et al. Hepatomegaly with severe steatosis in HIV-seropositive patients. AIDS 1993;7:379-85.

25 Mégarbane B, Brivet F, Guérin JM, et al. Acidose lactique et défaillance multi-viscérale secondaire aux thérapeutiques antirétrovirales chez les patients infectés par le VIH. Press Med 1999;28:2257-64.

26 Falcó V, Rodríguez D, Ribera E, et al. Severe nucleoside-associated lactic acidosis in human immunodeficiency virus-infected patients: report of 12 cases and review of the literature. Clin Infect Dis 2002;34:838-46.

27 Acosta BS, Grimsley EW. Zidovudine-associated type B lactic acidosis and hepatic steatosis in an HIV-infected patient. South Med J 1999:92:421-3

28 Miller KD, Cameron M, Wood LV, et al. Lactic acidosis and hepatic steatosis associated with use of stavudine: report of four cases. Ann Intern Med 2000;133: 192-6.

29 UNAIDS. Aids epidemic update: December 2000. UNAIDS/ 00.44E-WHO/CSR/EDC/2000.9

30 Bozzette SA, Berry SH, Duan N, et al. The care of HIV-infected adults in the United States. N Engl J Med 1998;339:1897-904.

31 Scalfaro P, Chesaux JJ, Buchwalder PA, et al. Severe transient neonatal lactic acidosis during prophylactic zidovudine treatment. Intensive Care Med 1998;24:247-50.

32 Blanche S, Tardieu M, Rustin P, et al. Persistent mitochondrial dysfunction and perinatal exposure to antiretroviral nucleoside analogues. Lancet 1999;354:1084-89.
33 Lenzo NP Garas BA, French MA. Hepatic steatosis and lactic acidosis associated with stavudine treatment in an HIV patient:a case report. AIDS 1997;11:1294-6.

34 Ter Hofstede HJM, de Marie S, Foudraine NA, et al. Clinical features and risk factors of lactic acidosis following long-term antiretroviral therapy: 4 fatal cases. Int J STD AIDS 2000;11:611-6.

35 Stenzel MS, Carpenter CCJ. The management of the clinical complications of antiretroviral therapy. Infect Dis Clin North Am 2000;14:851-78.

36 Carr A, Miller J, Cooper DA. A syndrome of lipoatrophy, lactic acidaemia and liver dysfunction associated with HIV nucleoside analogue therapy: contribution to protease inhibitor-related lipodystrophy syndrome. AIDS 2000;14:F25-32.

37 Lonergan T, Mccomsey G, Fisher R, et al. Improvements in symptomatic hyperlactatemia are observed after 12 weeks when stavudine is replaced by either abacavir or zidovudine. 3rd International Workshop on Adverse Drug Reactions and Lipodystrophy in HIV. Athens 2001 [abstract 81].

38 White AJ. Mitochondrial toxicity and HIV therapy. Sex Transm Infect 2001;77:158-73.

39 Walker UA, Stezer B, Venhoff N. Increased long-term mitochondrial toxicity in pyrimidine nucleoside combinations. 3rd International Workshop on Adverse Drug Reactions and Lipodystrophy in HIV. Athens 2001 [abstract 18].

40 Bartley PB, Westacott L, Boots RJ, et al. Large hepatic mitochondrial DNA deletions associated with L-lactic acidosis and highly active antiretroviral therapy. AIDS 2001;15:419-20.

41 Miller RF, Shahmanesh M, Hanna MG, et al. Polyphenotypic expression of mitochondrial toxicity caused by nucleoside reverse transcriptase inhibitors. Antiviral Ther (in press)

42 Masini A, Scotti C, Clligaro A, et al. Zidovudine-induced experimental myopathy: dual mechanism of mitochondrial damage. J Neurol Sci 1999; 166:131-40.

43 Leclercq $\mathbf{P}$, Roth $\mathrm{H}$, Bosseray $\mathrm{A}$, et al. Investigating lactate metabolism to estimate mitochondrial status. 3rd International Workshop on Adverse Drug Reactions and Lipodystrophy in HIV. Athens 2001 [abstract 21 ].

44 Brinkman K. Editorial response:hyperlactatemia and hepatic steatosis as features of mitochondrial toxicity of nucleoside analogue reverse transcriptase inhibitors. Clin Infect Dis 2000;31:167-9.

45 Lafeuillade A, Hittinger G, Chadapaud S. Increased mitochondrial toxicity with ribavirin in HIV/HCV coinfection. Lancet 2001;357:280-1.

\section{$\mathrm{ECHO}$}

\section{HAART homes in on lymphocyte mitochondria in lactic acidosis}

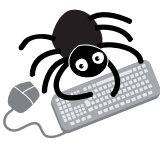

Please visit the Sexually Transmitted Infections website [www. stijournal.com] for link to this full article.
A severe adverse reaction to highly active antiretroviral treatment (HAART) has shown for the first time the treatment's potential for damaging lymphocyte mitochondria and increasing lymphocyte death. This has prompted speculation that cell death follows mitochondrial disruption.

The case of severe lactic acidosis occurred in a 39 year old woman who had been HIV positive for nearly 10 years and receiving HAART with stavudine, didanosine, and indinavir for just over two and a half years. Viral load was $<50$ copies/ml and CD4+ lymphocytes $>500 /$ $\mathrm{ml}$. When treatment was stopped serum lactic acid concentration was about $5 \mathrm{mmol} / \mathrm{l}$ and $\gamma$-glutathione 14 times higher than normal. Acidosis started to decrease only one month afterwards and returned to normal only two months later. Mitochondria in the lymphocytes dropped in number and those remaining appeared swollen and their internal structure disrupted, with loss of christae. These traits persisted for a month after treatment was withdrawn, during which time apoptosis of CD3 + lymphocytes peaked. Apoptosis and mitochondrial abnormalities gradually resolved as lactic acid concentration dropped, and despite an increased viral load of 21000 copies/ml. CD4+ lymphocyte numbers were unaffected.

Lymphocytes were typed by flow cytometry after staining with monoclonal antibodies. Apoptosis was measured by counting stained lymphocytes under fluorescence microscopy and by flow cytometry.

Lactic acidosis is a rare, but increasing, serious adverse reaction. This case provided a chance to see whether lymphocyte mitochondria are affected, given the importance of lymphocytes in HIV infection and reported mitochondrial damage in liver and skeletal muscle in similar cases.

A Journal of Clinical Pathology 2003;56:147-151. 